\title{
Selecting Control Parameters of Mechanical Systems with Servoconstraints
}

\author{
Kahramanjon Khusanov ${ }^{*}$ \\ Tashkent Institute of Irrigation and Agricultural Mechanization Engineers, Tashkent, Uzbekistan
}

\begin{abstract}
The research results on the derivation of equations of motion (a mathematical model) of a mechanical system constrained by holonomic constraints of the first and second kind, which contains only tangential components of the constraint of the second kind, are presented in the article. These tangential components are taken as control parameters. Besides, the controllability of the plate, considered in the Appel problem, is investigated.
\end{abstract}

\section{Introduction}

The study aims to identify the tangential components of the constraints of the second kind and consider them as a control influence.

Consider the motion of a mechanical system consisting of $N$ material points relative to some inertial Cartesian reference system $O x y z$.

Let $\boldsymbol{a}$ ideal constraints of the first kind be imposed on the system

$$
f_{\alpha}\left(x_{v}, t\right)=0, f \in c^{2} \quad(v=1,2, \ldots, 3 N ; \alpha=1,2, \ldots, a)
$$

and $c$ servoconstraints of the form [1]

$$
\varphi_{\rho}\left(x_{v}, t\right)=0, \quad \varphi_{\rho} \in c^{2}(\rho=1,2, \ldots, c)
$$

It is assumed that constraints (1) and (2) are independent, and their number satisfies the condition $a+c<3 N$. In addition, we consider that relations (1) refer to the N.G. Chetaev's class $[2,3]$ and variations of coordinates $\delta x_{v}$ are related $a+c$ by the following conditions

* Corresponding author : xusanov1954@gmail.com 


$$
\sum_{v} \frac{\partial f_{\alpha}}{\partial x_{v}} \cdot \delta x_{v}=0, \quad \sum_{v} \frac{\partial \varphi_{\beta}}{\partial \dot{x}_{v}} \cdot \delta x_{v}=0
$$

In what follows, we assume that servoconstraints are imperfect. Then the issue arises of the need to separate the normal and tangential components of the reactions of servoconstraints from the general reactions of constraints (2) since our goal is to control the system's motion under consideration with reactions of servoconstraints induced by servomotors. A servomotor is an unpretentious working element that is a part of industrial equipment. Servomotors have high-speed characteristics and high positioning accuracy. When properly operated, the servomotor is capable of operating 24 hours a day [4].

Modern servomotors have combined all the achievements of scientific and technical innovative progress; therefore, they can develop high rotational speed with very high power. A wide range of adjustments of the servomotor shaft rotation using software under significant acceleration or deceleration makes this equipment simply irreplaceable for use in machine tools or production lines and many other structures.

In [5-7], the movements of the system are investigated by the methods of the theory of elasticity, the theory of viscoelasticity, mathematical analysis, numerical methods, methods of mathematical modeling, algorithms and methods of the theory of systems with servoconstraints, under given displacements, on which the work of the reaction force of servoconstraints is zero, i.e., servoconstraints are ideal on these displacements.

The dynamics of systems with servo-constraints are discussed in [8] when the constraints are realized by controlling the inertial properties of the system. It is shown that the presence of symmetries allows reducing dynamic equations to a closed system of differential equations with quadratic right-hand sides.

In [9-11], mechanical systems are considered under the influence of various forces; nonlinear positional, potential, dissipative, gyroscopic forces, and the forces of radial correction. Gyroscopic forces are considered dominant, which is expressed by the presence of a large parameter-multiplier in the equations of motion. Conditions of the growth rate of the parameter were established; they guarantee the asymptotic stability of the equilibrium position both for a linear system and for essentially nonlinear dissipative forces given by a homogeneous Rayleigh function.

It is shown that for any values of the system's parameters (weight of loads, stiffness of springs), it is possible to provide asymptotic stability of the equilibrium position by attaching an additional load with a damper using a nonlinear spring. An approach to the study of stability and stabilization of mechanical systems with nonlinear positional forces proposed based on the decomposition of the equations of disturbed motion into two isolated subsystems. Conditions for stabilization of systems with nonlinear unsteady potential forces due to the forces of another structure are determined.

In [12-14], the results of studies on the control of the technological parameters in the process of extracting excess liquid from multilayer moisture-saturated fibrous materials (on the example of leather)are presented. Mathematical dependencies of the amount of extracted moisture for each layer of wet skin on their feeding rate, when supplied between the working shafts rotating at constant angular velocities and their squeezing pressure, were obtained.

However, in these studies, the explicit types of tangential and normal components of servoconstraints, which determine the number of control parameters, were not distinguished.

Let us designate the components of the reactions of constraints of the first and second kinds $N_{v}$ and $\Phi_{v}$, respectively. 


\section{Methods}

Consider the motion of a mechanical system consisting of $N$ material points relative to some inertial Cartesian reference system $O x y z$.

1. Let the constraints (1) and (2) be imposed on the system. Taking into account relations (3), we write down the generalized reactions of constraints (1) and (2) as:

$$
R_{v}=\sum_{\alpha} \lambda_{\alpha} \frac{\partial f_{\alpha}}{\partial x_{v}}+\sum_{\rho} \lambda_{\rho}^{\prime \prime} \frac{\partial \varphi_{\rho}}{\partial x_{v}}+\sum_{i} \mu_{i} \frac{\partial x_{v}}{\partial \widetilde{q}_{i}}
$$

where $\lambda_{\alpha}, \lambda_{\rho}^{\prime \prime}$ are the Lagrange multipliers, $\mu_{i}$ are certain coefficients.

Then the differential equation of motion has the form

$$
m_{v} \ddot{x}_{v}=X_{v}+\sum_{\alpha} \lambda_{\alpha} \frac{\partial f_{\alpha}}{\partial x_{v}}+\sum_{\rho} \lambda_{\rho}^{\prime \prime} \frac{\partial \varphi_{\rho}}{\partial x_{v}}+\sum_{i} \mu_{i} \frac{\partial x_{v}}{\partial \widetilde{q}_{i}}
$$

Let us multiply each of the equations (4) by $\delta x_{v}$ and sum up over $v$ from 1 to $3 N$.

Taking into account the variations $\delta x_{v}=\sum_{i} \frac{\partial x_{v}}{\partial \widetilde{q}_{i}} \cdot \partial \widetilde{q}_{i}$, we obtain [15]

$$
\sum_{i}\left[\frac{d}{d t} \frac{\partial T}{\partial \dot{\vec{q}}_{i}}-\frac{\partial T}{\partial \widetilde{q}_{i}}-Q_{i}-\sum_{\alpha} \lambda_{\alpha} \frac{\partial \widetilde{f}_{\alpha}}{\partial \widetilde{q}_{i}}-\sum_{\tau} \mu_{\tau} A_{i \tau}\right] \cdot \delta \widetilde{q}_{i}=0
$$

where

$$
T=T\left(\widetilde{q}_{i}, \dot{\widetilde{q}}_{i}, t\right), Q_{i}=\sum_{v} X_{v} \frac{\partial x_{v}}{\partial \widetilde{q}_{i}}, \quad A_{i \tau}=\sum_{v} \frac{\partial x_{v}}{\partial \widetilde{q}_{1}} \cdot \frac{\partial x_{v}}{\partial \widetilde{q}_{\tau}}, \quad \frac{\partial \tilde{f}_{\alpha}}{\partial \widetilde{q}_{i}}=\sum_{v} \frac{\partial f_{\alpha}}{\partial x_{v}} \cdot \frac{\partial x_{v}}{\partial \widetilde{q}_{i}} .
$$

Let equations of constraints (1), expressed in terms of generalized coordinates $\widetilde{q}_{1}, \widetilde{q}_{2}, \ldots, \vec{q}_{S}$, be satisfied by the relations

$$
\widetilde{q}_{i}=\widetilde{q}_{i}\left(q_{1}, q_{2}, \ldots, q_{S}\right) ; \quad(i=1,2, \ldots, n ; s=n-a)
$$

Writing down the last relations in variations and substituting them into equations (5), we determine

$$
\sum_{j}\left[\frac{d}{d t} \frac{\partial \widetilde{T}}{\partial \dot{q}_{j}}-\frac{\partial \widetilde{T}}{\partial q_{j}}-\widetilde{Q}_{j}-\sum_{\tau} \mu_{\tau} a_{j \tau}\right] \delta q_{j}=0
$$

From that follows the system of differential equations

$$
\frac{d}{d t} \frac{\partial \widetilde{T}}{\partial \dot{q}_{j}}-\frac{\partial \widetilde{T}}{\partial q_{j}}=\widetilde{Q}_{j}+\sum_{\tau} \mu_{\tau} a_{j \tau}
$$


Here

$\widetilde{T}=\widetilde{T}\left(q_{j}, \dot{q}_{j}, t\right)$ is the kinetic energy of the system;

$\widetilde{Q}_{j}=\sum_{i} Q_{i} \frac{\partial \widetilde{q}_{i}}{\partial q_{j}}$ are the generalized forces referred to coordinates $q_{j}$

$a_{j \tau}=\sum_{i} A_{i \tau} \frac{\partial \widetilde{q}_{i}}{\partial q_{j}}$ are the known functions of time and generalized coordinates $q_{j}$.

Equation (6) contains only tangential components of the reactions of servoconstraints (2). Let us write equation (6) in the expanded form [16, 17]; assuming that the expression for kinetic energy has the following form

$$
\widetilde{T}=\frac{1}{2} \sum_{j} \sum_{\ell} A_{j \ell} \dot{q}_{j} \dot{q}_{\ell}+\sum_{j} B_{j} \dot{q}_{j}+T_{0}^{*}
$$

we obtain

$$
\begin{gathered}
\sum_{\ell} A_{\ell j} \ddot{q}_{\ell}+\sum_{\ell} \sum_{m}[\ell, m ; j]_{q} \dot{q}_{\ell} \dot{q}_{m}=\Gamma_{j}+\widetilde{Q}_{j}-\frac{\partial \widetilde{T}_{0}}{\partial q_{j}}-\frac{\partial B_{j}}{\partial t}-\sum_{\ell} \frac{\partial A_{\ell j}}{\partial t} \dot{q}_{\ell}+\sum_{\tau} \mu_{\tau} a_{j \tau}(7) \\
{[\ell, m ; j]_{q}=\frac{1}{2}\left[\frac{\partial A_{\ell j}}{\partial q_{m}}+\frac{\partial A_{m j}}{\partial q_{\ell}}-\frac{\partial A_{\ell m}}{\partial q_{j}}\right], \Gamma_{j}=\sum_{\ell} g_{j \ell} \dot{q}_{\ell}, \quad g_{j l}=\frac{\partial B_{\ell}}{\partial q_{j}}-\frac{\partial B_{j}}{\partial q_{\ell}}}
\end{gathered}
$$

In the case of stationary constraints, we obtain

$$
\sum_{\ell} A_{e j} \ddot{q}_{\ell}+\sum_{\ell} \sum_{m}[\ell, m ; j]_{q} \dot{q}_{\ell} \dot{q}_{m}=\widetilde{Q}_{j}+\sum_{\tau} \mu_{\tau} a_{j \tau}
$$

Solving equations (7) and (8) with respect to $\ddot{q}_{j}$ we find

Where

$$
\begin{gathered}
\ddot{q}_{r}+\sum_{\ell} \sum_{m}\left\{\begin{array}{c}
r \\
\ell, m
\end{array}\right\} \dot{q}_{\ell} \dot{q}_{m}=\sum_{j} A_{j r}^{-1}\left(\Gamma_{j}+\widetilde{Q}_{j}-\frac{\partial \widetilde{T}_{0}}{\partial q_{j}}-\frac{\partial B_{j}}{\partial t}-\sum_{\ell} \frac{\partial A_{\ell j}}{\partial t} \dot{q}_{\ell}+\sum_{\tau} \mu_{\tau} a_{j \tau}\right) \\
\ddot{q}_{j}+\sum_{\ell} \sum_{m}\left\{\begin{array}{c}
j \\
\ell, m
\end{array}\right\} \dot{q}_{\ell} \dot{q}_{m}=\sum_{\ell} A_{j \ell}^{-1}\left(\widetilde{Q}_{j}+\sum_{\tau} \mu_{\tau} a_{j \tau}\right)
\end{gathered}
$$

$\left\{\begin{array}{c}r \\ \ell, m\end{array}\right\}=\sum_{j} A_{j r}^{-1}[\ell, m ; j]$ are the Christoffel symbols of the second kind 


\section{Results and Discussion}

Now let us consider some special cases.

If it is required to consider the normal components of the reactions of servoconstraints

(2), then it is advisable to introduce generalized coordinates taking into account constraints (1).

$$
\begin{gathered}
x_{v}=x_{v}\left(y_{1}, y_{2}, \ldots, y_{\ell}, t\right) \\
\delta x_{v}=\sum_{r} \frac{\partial x_{v}}{\partial y_{r}} \delta y_{r}(r=1,2, \ldots, \ell ; \ell=3 N-a)
\end{gathered}
$$

Then equation (4) can be reduced to the form

$$
\frac{d}{d t} \frac{\partial T}{\partial \dot{y}_{r}}-\frac{\partial T}{\partial y_{r}}=Q_{r}+\sum_{\rho} \lambda_{\rho}^{\prime \prime} B_{\rho r}+\sum_{i} \mu_{i} A_{i r}
$$

Where

$$
\begin{gathered}
T=T\left(y_{1}, y_{2}, \ldots, y_{\ell}, \dot{y}_{1}, \dot{y}_{2}, \ldots, \dot{y}_{\ell}, t\right) \\
Q_{r}=\sum_{v} X_{v} \frac{\partial x_{v}}{\partial y_{r}} ; \quad B_{\rho r}=\sum_{v} \frac{\partial \varphi_{\rho}}{\partial x_{v}} \frac{\partial x_{v}}{\partial y_{r}} ; \quad A_{i r}=\sum_{v} \frac{\partial x_{v}}{\partial \widetilde{q}_{i}} \frac{\partial x_{v}}{\partial y_{r}}
\end{gathered}
$$

These equations are coupled with equations of constraints (2) expressed in generalized coordinates $y_{1}, y_{2}, \ldots, y_{\ell}$, i.e.

$$
\widetilde{\varphi}_{\rho}\left(y_{1}, y_{2}, y_{3}, \ldots, y_{\ell}, t\right)=0
$$

If a system for which constraints of the first kind have already been taken into account is additionally superimposed with constraints of the form

$$
\varphi_{\rho}^{*}\left(\widetilde{q}_{1}, \widetilde{q}_{2}, \ldots, \widetilde{q}_{S}, t\right)=0 ; \quad(\rho=1,2, \ldots, c)
$$

then, instead of equations (6), we should use the following equations:

$$
\frac{d}{d t} \frac{\partial T^{*}}{\partial \dot{q}_{m}}-\frac{\partial T^{*}}{\partial q_{m}}=Q_{m}^{*}+\sum_{j} \mu_{j}\left(\sum_{i} \frac{\partial \widetilde{q}_{i}}{\partial q_{m}} \frac{\partial \widetilde{q}_{i}}{\partial q_{j}}\right),
$$

where $T^{*}$ is the kinetic energy of the system, determined with an account of (9).

If we introduce the notation

$$
P_{j}=\frac{\partial \widetilde{T}}{\partial \dot{q}_{j}}, \quad \Phi=\sum_{j} p_{j} \dot{q}_{j}-\widetilde{T}
$$


then equation (6) can be written in the form of Hamilton's equations [16] (here, the velocities $\dot{q}_{j}$ should be expressed in terms of the moment)

$$
\frac{d p_{j}}{d t}=-\frac{\partial \Phi}{\partial q_{j}}+\widehat{Q}_{j}+\sum_{\tau} \widehat{\mu}_{\tau} a_{j r}, \quad \frac{d q_{j}}{d t}=\frac{\partial \Phi}{\partial p_{j}}
$$

and we obtain $2 n$ equations of the first order concerning $3 n$ unknown functions of time $q_{j}, p_{j}$ and $\mu_{\tau}$. When studying the motion of systems with friction, in addition to the given forces, it is necessary to consider known expressions for the coefficients $\mu_{\tau}$ depending on $\lambda_{\rho}^{\prime \prime}, q_{j}, \dot{q}_{j}, t$; the form of these functions is found empirically [18]. If the values $\mu_{\tau}$ are not set in advance, then the resulting uncertainty can be useful when considering optimal (in one way or another) modes of motion [19].

As an example of the application of equation (10), consider the problem of the plane motion of a plate hinged to a circular disc $[1,15]$.

The plate $\Sigma$, located on a fixed horizontal plane, is hinged at point $C$ with a circular disc $\Sigma_{1}$ lying in the same plane and moving around its fixed center $O$. Constant force $\vec{F}$ parallel to a fixed straight line $O x$ acts on the plate at a point $A$ lying on a straight line connecting the point $C$ with the center of gravity $G$. The servomotor $\mathrm{M}$ with a special coupling makes direct contact between $\Sigma$ and $\Sigma_{1}$ so that the following constraint is constantly realized

$$
\begin{gathered}
\alpha-\beta=\frac{\pi}{2} \\
{[\alpha=(\hat{O x, O C}), \beta=(O \hat{x}, C A), O C=R, C \mathrm{~A}=a, C \mathrm{G}=b]}
\end{gathered}
$$

Suppose that servo-constraint (11) is realized in a way in which the response of the servo-constraint is non-zero on virtual displacements determined by the following condition

$$
\delta \alpha-\delta \beta=0
$$

The kinetic energy of the system is

$$
T=\frac{1}{2} M\left[R^{2} \dot{\alpha}^{2}+b^{2} \dot{\beta}^{2}+2 R b \cos (\alpha-\beta) \dot{\alpha} \dot{\beta}+k^{2} \dot{\beta}^{2}\right]+\frac{1}{2} J_{1} \dot{\alpha}^{2}
$$

where $J_{1}$ is the moment of inertia of the disc relative to the point $O$, and the virtual work of a constant force $\vec{F}$ is

$$
\delta A=F \delta(R \cos \alpha+a \cos \beta)
$$


We take angle $\beta$ as a generalized coordinate, i.e.

$$
q_{1}=\beta\left(\widetilde{q}_{1}=\beta, \widetilde{q}_{2}=\alpha\right)
$$

Let us introduce a function $\widetilde{q}_{2}=q_{1}+\pi / 2$ that identically satisfies the constraint equation (11). Then the equation of motion can be written in the following form (10)

$$
\frac{d}{d t} \frac{\partial T^{*}}{\partial \dot{q}_{1}}-\frac{\partial T^{*}}{\partial q_{1}}=Q_{1}^{*}+\mu\left(\frac{\partial \widetilde{q}_{1}}{\partial q_{1}} \frac{\partial \widetilde{q}_{1}}{\partial q_{1}}+\frac{\partial \widetilde{q}_{2}}{\partial q_{1}} \frac{\partial \widetilde{q}_{2}}{\partial q_{1}}\right)
$$

or

$$
\ddot{q}_{1}\left[M\left(R^{2}+b^{2}+k^{2}\right)+J_{1}\right]+F\left(R \cos q_{1}+a \sin q_{1}\right)=2 \mu
$$

If the servomotor was installed on a disc $\Sigma_{1}$, then the differential equation of motion of the system could be written in the form:

$$
\left.\ddot{q}_{1}\left[M\left(b^{2}+k^{2}\right)\right]-M R b \dot{q}_{1}^{2}+F a \sin q_{1}\right)=0
$$

Let equation (12) allow for a particular solution in the form $q_{1}=\frac{\pi}{2}, \mu^{0}=\frac{F a}{2}$.

Assuming the disturbance

we obtain

$$
q_{1}=\frac{\pi}{2}+x, \quad \mu^{0}=\frac{F a}{2}+u
$$

$$
\left[M\left(R^{2}+b^{2}+k^{2}\right)+J_{1}\right] \ddot{x}+F R x=2 u+\Phi,
$$

where $\Phi$ are the second-order nonlinear terms.

Denoting $x=y_{1}, \dot{y}_{1}=y_{2}$, we obtain

$$
\dot{y}=P y+Q u
$$

where

$$
P=\left\|\begin{array}{cc}
0 & 1 \\
\frac{F R}{M\left(R^{2}+b^{2}+k^{2}\right)+J_{1}} & 0
\end{array}\right\|, \quad Q=\| \frac{0}{2}
$$

Following [20], we compose the matrix 


$$
W=[Q, P Q]=\left\|\begin{array}{cc}
0 & \frac{2}{M\left(R^{2}+b^{2}+k^{2}\right)+J_{1}} \\
2 & 0
\end{array}\right\| \neq 0,
$$

i.e., the system is completely controllable with the tangential component of the servoconstraint reaction.

Let the quality of the transient process in system (13) be estimated by the functional

$$
J=\int_{0}^{\infty}\left(y_{1}^{2}+y_{2}^{2}+u^{2}\right) d t
$$

Then the optimal Lyapunov function $V^{0}\left(t, y_{1}, y_{2}\right)$ is sought in the form [20-22]

$$
V^{0}=c_{11} y_{1}^{2}+2 c_{12} y_{1} y_{2}+c_{22} y_{2}^{2}
$$

To determine coefficients $\boldsymbol{C}_{i j}$, we obtain the equations

$$
\begin{gathered}
2 A c_{21}-B^{2} c_{21}^{2}+1=0,2 c_{12}-B^{2} c_{22}^{2}+1=0, \\
c_{11}+A c_{22}-B^{2} c_{21} c_{12}=0 ; \quad c_{12}=c_{21},
\end{gathered}
$$

Where

$$
A=-\frac{R F}{M\left(R^{2}+b^{2}+k^{2}\right)+J_{1}}, \quad B=\frac{2}{M\left(R^{2}+b^{2}+k^{2}\right)+J_{1}} .
$$

The values of $c_{i j}(i, j=1,2)$ are obtained as

$$
\begin{gathered}
c_{11}=\frac{\sqrt{B^{2}+2 A+2 \sqrt{A^{2}+B^{2}} \sqrt{A^{2}+B^{2}}}, \quad c_{12}}{B^{3}}=\frac{A+\sqrt{A^{2}+B^{2}}}{B^{2}}, \\
c_{22}=\frac{\sqrt{B^{2}+2 A+2 \sqrt{A^{2}+B^{2}}}}{B^{3}} .
\end{gathered}
$$

The desired optimal control $u^{0}$ is determined by the formula [21]

$$
u^{0}=\frac{1}{2}\left[\frac{\partial V^{0}}{\partial y_{1}} q_{11}+\frac{\partial V^{0}}{\partial y_{2}} q_{21}\right]=-B\left(c_{12} y_{1}+c_{22} y_{2}\right)
$$

The dynamics of the transient process of a closed system, that is, the time changes of the coordinates of a dynamic system, up to a certain steady-state, is 


$$
\dot{y}_{1}=y_{2} ; \quad \dot{y}_{2}=-A y_{1}-B^{2}\left(c_{12} y_{1}+c_{22} y_{2}\right)
$$

$\frac{d V^{0}}{d t}$ is a negative definite function. Therefore, the impact $u^{0}$ provides asymptotic stability of undisturbed motion.

\section{Conclusions}

Considering the P. Painlevé constraint-based method, the equations of motion of mechanical systems with ideal and non-ideal holonomic constraints, containing only tangential components of non-ideal constraints, were derived. These tangential components of the servoconstraints were proposed as control parameters.

The equations of motion obtained are given in the form of the Lagrange and Hamilton equations. A plate hinged to a circular disc is considered an example. Unlike A. Beguin, it is assumed that servomotor forces act on the hinge. The equations of the disturbed motion were derived, and the optimal control was determined. It was shown that the tangential components of the servoconstraints of a plate hinged to a circular disc are quite controllable.

\section{References}

1. Begen A. Theory of gyroscopic compasses, p. 192, Moscow, Nauka, (1967)

2. Azizov A.G. Questions of analytical dynamics of systems with servoconstraints: Phys.-Math. Sci. - M., p. 220, (1974)

3. Azizov A.G. On one method of realizing servoconstraints imposed on a mechanical system, Izv. AS UzSSR, Ser. Tech. Sci. № 3. pp. 27-31, (1972)

4. Zelectro C.C. What is servo - Device, principle of operation, characteristics and dimensions of servo drives, (2013)

5. Teshaev M.Kh. Searching the structure of the reaction forces of servoconstraints in systems constrained by geometrical constraints, Problems of Mechanics and Control. Nonlinear dynamical systems, Interuniversity Collection of Scientific Papers, 47, pp.97-104 (2015)

6. Teshaev M.K.Realization of servo constraints by electromechanical servosystems. Russian Mathematics, 54(12), pp. 38-44, (2010)

7. Mirsaidov M., Teshaev M., Ablokulov, S., Rayimov, D. Choice of optimum extinguishers parameters for a dissipative mechanical system, IOP Conference Series: Materials Science and Engineering, 883(1), (2020)

8. Kozlov V.V. Dynamics of systems with servoconstraints, II, Nonlinear Dynamics, 11(3), pp. 579-611, (2015)

9. Kozlov V.V. The dynamics of systems with servoconstraints. II, Regular and Chaotic Dynamics, 20(4), pp. 401-427, (2015)

10. Aleksandrov A.Y., Zhabko A.P., Kosov A.A. Analysis of stability and stabilization of nonlinear systems based on decomposition, Siberian Mathematical Journal, 56(6), pp. 1215-1233, (2015)

11. Aleksandrov A.Y., Aleksandrova E.B. Asymptotic stability conditions for a class of hybrid mechanical systems with switched nonlinear positional forces, Nonlinear Dynamics, 83, (4), pp. 2427-2434, (2016)

12. Alexandrov A.Yu., Kosov A.A. On the stability of gyroscopic systems, Bulletin of St. Petersburg University, ser. 10(2). Applied Mathematics, (2013) 
13. Amanov A, Bahadirov G, Amanov T, Tsoy G and Nabiev A. Determination of Strain Properties of the Leather Semi-Finished Product and Moisture-Removing Materials of Compression Rolls Materials (Basel).12

14. Bahadirov G., Tsoy G., and Nabiev A., "Study of the efficiency of squeezing moisture-saturated products," EUREKA Phys. Eng., (2021)

15. Amanov A., Bahadirov G., Tsoy Gerasim, Nabiev A. M. A New Method to Wring Water-Saturated Fibrous Materials, Int. J. Mech. Eng. Robot. Res., 10(3), pp. 151156, (2021)

16. Appel P. Theoretical Mechanics. System dynamics, Analytical Mechanics: Translated from French, 2, p. 487, Moscow, (1960)

17. Suslov G.K. Theoretical mechanics, p. 654, Moscow, (1946)

18. Rumyantsev V.V. On systems with friction // Applied Mathematics and Mechanics, 25(6), pp. 969-977, (1961)

19. Painlevé P. Lectures on friction, p. 316, Moscow, (1954)

20. Apykhtin N.G., Yakovlev V.F. On the motion of dynamically controlled systems with variable masses, Applied Mathematics and Mechanics, 44(3), pp. 427-433, (1980)

21. Krasovsky N.N. Motion control theory, p. 475, Moscow, Nauka, (1968)

22. Malkin I.G. The theory of motion stability, p. 530, Moscow, (1966)

23. Gabrielyan M.S. Stabilization of unstable motions of mechanical systems, Applied Mathematics and Mechanics, 28(3), pp. 493-501, (1964) 